\title{
Reconciling complexity and classification in quality improvement research
}

\author{
Laura Leviton
}

Correspondence to Ms Laura C Leviton, The Robert Wood Johnson Foundation, Route 1 and College Road East, Box 2316 , Princeton, NJ 08543-2316, USA; Ilevito@rwjf.org

Accepted 24 November 2010

\begin{abstract}
Quality improvement (QI) research is often hampered by the complexity of the systems and context in which $\mathrm{Q}$ is attempted. Better classification of $\mathrm{QI}$ can alleviate this problem and help to build generalisable knowledge.
\end{abstract}

For many quality improvement (QI) practitioners, both the challenge and the joy is that QI occurs in complex adaptive systems. The unexpected can and does happen, and practitioners need to be free to use their ingenuity to address QI problems. At the same time, complexity often defies researchers, who need to create generalisable knowledge about QI within reasonably well-specified parameters. If the hallmarks of QI are unpredictability and variety, then how can we study or report on dependable interventions for effective QI that could be shared with others? On the other hand, if we believe generalisable knowledge is important, how can we avoid 'freezing' and oversimplifying the QI practice context?

This tension is shared with other fields. Practitioners often feel constrained by research findings that do not acknowledge complexity and the need for adaptation, and where practitioner knowledge has been subordinated to research findings. ${ }^{1}$ This brief commentary aims to provoke questions about this shared dilemma and suggest a potential way forward.

In fact, complex contexts do reveal patterns; it is just that the patterns often go unrecognised. Better classification of QI interventions could help. QI has plenty of classification tools, ${ }^{2}$ but they do not necessarily describe the principles underlying implementation of a common approach to a QI problem. Such QI principles are important for testing effectiveness, for theory building and for developing sorely needed adaptations of QI interventions in a local context. Without them, how will we better specify the 'it' that is claimed to be effective? How can we make sense of QI efforts in their larger context (theory) or generalise claims about QI for new problems and settings?

Classifying phenomena is an important scientific activity, and it often precedes theory and generalisation. For example, Darwin developed the theory of evolution in large part because of the naturalists' work on classification of living creatures. ${ }^{3}$ In truth, the classification helped to drive the development of the theory. In social science, this process is called pattern matching; it is a critical support both for theory building and tests of social programme effectiveness. ${ }^{4}$

Part of the problem for QI is that important features of implementation and context go unreported, so no patterns can be recognised. For this, the SQUIRE guidelines should help. ${ }^{5}$ But to recognise a pattern, we need a large number of relevant examples. How can we best go from a single case to a systematic recognition of patterns? Like the early naturalists, we first need to collect and study many variants of QI that we believe are important. Otherwise, how can we tell the hummingbirds from the ducks? We need a more abundant description of organisational case studies, ${ }^{6}$ as well as surveys, for example of the role of hospital infrastructure in QI. ${ }^{7}$ Only by collecting, comparing and analysing such cases will it be possible to identify the essential elements of QI and its context, classify and test them, and move theory along. Methods have long been available to analyse complex case studies. ${ }^{8}$ Studying cases in this way does not reduce their importance-on the contrary, it elevates their importance for research.

However, QI is dynamic and ever-changing, so the analogy to evolutionary theory only 
goes so far. It is as though Darwin were trying to classify species with the evolutionary process speeded up a thousand-fold. We dare not treat QI as static classification, or rely exclusively on retrospective case studies. Instead, QI research needs to develop dynamic models of context, ${ }^{9} 10$ and it needs a capacity for real-time reflection about the emerging patterns.

The field might be assisted by tapping more systematically into what practitioners know. The literature on reflective practice may assist us. ${ }^{1}$ Reflective practitioners (in any field) have seen a great many situations and have developed a repertoire of solutions. They draw on this repertoire to deal with new or puzzling situations-in other words, complexity. Reflective practitioners then assess the results and incorporate the experience into their repertoires, along with any theory and empirical evidence that their field may possess.

QI practitioners follow this pattern to some degree, even though they are a heterogeneous group. The QI field is still emerging, still relies a great deal on trial and error, and lacks a strong theory and empirical base. Nevertheless, we might expect that experienced QI practitioners could help researchers to see the larger patterns at work in complex systems and draw upon potential solutions or generalised principles. QI practitioners could serve as 'participant observers' for QI research, and in that role they could contribute first-hand, on-the-ground reports of 'what really goes on in QI projects'.

This process cannot work without guidance, however. To ensure that QI practitioners reported clear, useful and coherent information, researchers would need to train them in much the same way that observers are trained in a rapid ethnographic method. ${ }^{11}$ At the same time, reflective practitioners might 'train' the researchers by helping them to better define their terms! The paper by Øvretveit, Leviton and Parry in this issue outlines one approach to doing so. ${ }^{12}$
This approach is compatible with many of the calls for change in pedagogy, practice and QI methods throughout this special journal issue. All of them help to embed QI research in a community of practice. ${ }^{13}$ Like Darwin, the QI field is launched on its own voyage, but we have to believe that the sea of complex healthcare will still allow for generalisable knowledge.

Acknowledgements Thanks to an anonymous reviewer and to the editors for some of the language used in this commentary.

Competing interests None.

Provenance and peer review Not commissioned; externally peer reviewed.

\section{REFERENCES}

1. Schön DA. The Reflective Practitioner: How Professionals Think in Action. New York: Basic Books, 1984.

2. Institute of Medicine, Committee on Quality of Health Care in America. Crossing the Quality Chasm: A New Health System for the $21^{\text {st }}$ Century. Washington, DC: National Academies Press, 2001.

3. van Whye J. Charles Darwin: Gentleman naturalist. In The Complete Work of Charles Darwin Online 2010. http://darwin-online.org.uk/ darwin.html.

4. Trochim W. Pattern matching, validity and conceptualization in program evaluation. Eval Rev 1985;9:575-604.

5. Davidoff F, Batalden P, Stevens D, et al. Publication guidelines for quality improvement in health care: evolution of the SQUIRE project. Qual Saf Health Care 2008;17(Suppl 1):i3-i9.

6. Baker GR. The contribution of case study research to knowledge of how to improve quality of care. BMJ Qual Saf 2011;20(S1): i30-i35.

7. Alexander JA, Weiner BJ, Shortell SM, et al. The role of organizational infrastructure in implementation of hospitals' quality improvement. Hosp Top 2006;84:11-20.

8. Yin RK, Heald KA. Using the case survey method to analyze policy studies. Admin Sci Quart 1975;20:371-81.

9. Rogers PJ. Using programme theory to evaluate complicated and complex aspects of interventions. Evaluation 2008;14:29-48.

10. Greenhalgh T, Stones R. Theorising big IT programmes in healthcare: strong structuration theory meets actor-network theory. Soc Sci Med 2010;70:1285-94.

11. Scrimshaw S, Hurtado E. Rapid Assessment Procedures for Nutrition and Primary Health Care: Anthropological Approaches to Improving Programme Effectiveness. Los Angeles: University of California Press, 1987.

12. Øvretveit J, Leviton L, Parry G. Increasing the generalisability of improvement research with an improvement replication programme. BMJ Qual Saf 2011;20(S1):i87-i91.

13. Wenger E. Communities of practice: a brief introduction. http://www. ewenger.com/theory/. 